months. It has also been demonstrated in Case 1 that radium has had a very decided effect in reducing the size of a metastatic growth associated with carcinoma of the bladder.

3. No difference seems to have been evident in the result whether or not part of the tumor was removed. In Case 1 no part of the tumor was removed, while in Case 2 a considerable portion of the tumors was excised.

4. We are of the opinion that the peculiar pyrexia associated in both cases was due to an absorption following the destructive influence of the radium on the tumors. A thorough investigation did not disclose any other cause.

We wish to take this opportunity of thanking Dr. Wolf Freudenthal for the use of the radium applied in these cases.

10 West Sixty-First Street-1847 Madison Avenue.

\section{A CASE OF BACILLUS DYSENTERIAE SEPTICEMIA}

W. T. De Sautelle, M.D., Knoxville, Tenn.

In the literature at my disposal, instances of isolation of the Bacillus dyscnteriae from the blood during life seem to be very few in number. Darling and Bates (Jour. Med. Sc., 1912, cxliii, 36), report a fatal case from which they recovered from the circulating blood the $B$. dysenteriae of the Shiga type. All of the references at hand either make no mention of the $B$. dy'senteriac entering the general circulation or state positively that it does not do so. It seemed to be of interest therefore to report the following case which was seen in consultation with Dr. C. G. Hutson of this city.

The patient was a 3 -weeks-old breast-fed infant which had been in perfect health before the onset of the disease. For a day or two the baby refused to nurse, became irritable, restless and did not sleep. The child was stupid and listless, whined at times and objected to the slightest touch. Physical examination revealed no abnormalities or pathologic processes in any of the organs. The temperature was 103 and pulse 180 . At this time there was no diarrhea. In consideration of the fact that the grandmother of the infant was suffering in the same house with typhoid fever, a blood-culture was made. As we were unable to enter a vein, the blood was taken from the great toe and placed in bouillon from which the B. dy'senteriae was obtained in pure culture. Although the baby's toe was carefully cleansed and the blood dropped directly into the bouillon, we did not give much significance to the result of the culture.

The baby continued to have a fever varying from 101 to 104.5 in spite of antipyretic measures and also developed a watery diarrhea. Five days later the baby suddenly began to have severe convulsions which came on every few minutes and lasted from five to ten minutes. When seen the hands, face and fect were very cyanotic, the pulse thirty to the minute, the breathing of the Cheyne-Stokes type, the pupils dilated and inactive to light, divergent strabismus, the tendon reflexes absent (at the first examination they were normal), and the child comatose. Through a lumbar puncture needle the spinal fluid flowed out quite rapidly, but contained at least one-half blood. The fluid came as bloody at the end of the flow as at the beginning, in fact, the needle was withdrawn and reintroduced at three different places with the same result. After the removal of 3 or 4 c.c. spinal fluid the convulsions ceased for a time, the pulse and respiration improved, and the cyanosis cleared up. The child died several hours later. Post-mortem was refused.

The spinal fluid obtained was carefully placed in bouillon and an organism found which was identical in all respects with the one obtained in the blood-culture.

The organism cultivated from the blood and spinal fluid had the following characteristics: It was a Gram-negative short bacillus, only sluggishly motile, and did not produce indol in five days or coagulate or digest milk. It produced acid in litmus milk and this remained so for three days. In dextrose, levulose, lactose, mannite, galactose, maltose and dextrin bouillon this bacillus produced acid but no gas in twenty-four hours. Saccharose and inulin were unchanged. At the end of three days the mediums remained unchanged.

The organism corresponding to these reactions is the bacillus "Rosen" (from dysentery) and we therefore concluded that this was a case of Bacillus dysenteriae septicemia. The finding of the same organism in the bloody cerebrospinal fluid and the blood taken from the child's toe convinced us that the organism had been present in the general circulation from the onset of the illness. Death was no doubt due to the cerebral hemorrhage rather than to the toxemia. Nevertheless this case with the fatal case previously mentioned would lead one to think that the advent of the $B$. dysenteriae into the general circulation means a fatal outcome of such cases. More reports of Bacillus dysenteriae septicemia are necessary, to be sure, before drawing such a conclusion.

The source of infection could not be ascertained. The child was breast fed and the parents denied having given it any other food. It may have been given unboiled water, but this they also denied. The sick grandmother handled the infant and even kissed it on the mouth several times. The diagnosis in her case, however, was unquestionably typhoid fever, as a blood-culture done by Dr. A. L. Snith in our laboratory revealed an organism identical in all its reactions to the Bacillus typhosus.

417 Holston Bank Building.

\section{HYGROMA OF THE GROIN}

Percy Willard Roberts, M.D., New York

The following case of hygroma in the groin is of interest on account of its apparent rarity and also because the method by which healing was finally induced is not commonly in vogue.

Surgical literature contains very little concerning hygroma in the groin. In a carefully prepared paper by Dowd in the Annals of Surgery for June, 1913, in which are reported several of his own cases, he goes over the literature of hygroma and refers to the fact that it seldom appears in the groin. A rather painstaking search fails to reveal any reported instances of cystic formations in this region, though, doubtless, cases have been noted, for the abundant supply of lymphatic tissue here would indicate a fertile field for their development.

The patient was a boy of 16 who was sent me with a diagnosis of psoas abscess, to which clinical appearances pointed. There was present at the upper and anterior aspect of the right thigh a large tumor extending from the groin half way to the knee, with the characteristic feel of a tense abscess. On the outer side of the thigh there was a discharging sinus through which a probe could be passed to a point over the head of the femur. Examinations of the hip-joint and spine were, however, negative, as were also roentgenograms of these parts.

Nevertheless, the clinical picture was one of cold abscess. The swelling had enlarged rapidly, having developed in three months without pain or temperature, and there was no history of injury or infection.

The patient was taken to the Post-Graduate Hospital, and incision of the tumor resulted in the evacuation of about a quart of a clear, straw-colored fluid which contained no pathogenic organisms. Exploration revealed a multilocular cavity extending to the ramus of the ischium without definite source of origin, the bone not being involved. There was no connection with the sinus on the outer side of the thigh. The cavity was lined with an extremely delicate membrane, but there was apparently no limiting membrane of a fibrous nature, the resistance of the muscles and fascia acting as a retaining wall.

The cavity was thoroughly scraped and allowed to drain. A few days later a constriction half way toward the ischium closed and the upper end of the cyst rapidly refilled, neces- 
sitating a second opening, which was followed by scraping and the application of iodin. The sinus on the outer side of the thigh, which was probably the remains of a ruptured cyst, was also thoroughly scraped. Both cavities were treated with iodin, phenol (carbolic acid), bismuth and argyrol over a period of fourteen weeks without any attempt at healing from the bottom, although both contracted in circumference. There was still a free secretion of clear fluid.

In view of the effect of the Roentgen ray on old tuberculous sinuses, many of which will heal under its influence, the patient was given a four-minute exposure with a hard tube. At the end of ten days both sinuses had begun to fill in at the bottom. Three other similar exposures at intervals of ten days completed the cure.

The laboratory report on the scrapings removed from the cavity was not conclusive, owing, no doubt, to the fact that the specimen was taken at the second operation and did not include enough of the lining membrane to determine its exact nature. It would seem, however, that from the delicacy of the lining membrane, the multilocular character of the cyst and the rapidity and force of its growth, the case should properly be classified under the head of hygroma.

40 East Forty-First Street.

\section{Therapeutics}

\section{PREVENTION IS GREATER THAN CURE \\ (Continued from page 1761)}

IX

EUGENICS

This very large social, economic, medical, religious and legal subject should receive a passing thought in this series on preventive medicine.

Briefly, heredity and environment are the two factors that are most prominent in the production of physical and mental health. Environment may improve or mar heredity, but cannot change it. Heredity is therefore the most important factor in raising and developing an ideal race. Scientific selection in breeding animals and plants has proved its value beyond all possible refutation. The importance of good environment for the perpetuation of physical and mental health is so well understood that it requires no discussion. But environment will not eliminate a hereditary tendency to disease or to mental or physical insufficiency. Neither will environment develop perfect mental and physical health when there is an inherited deficiency, although environment can markedly improve deficiency caused by injury or acquired by disease.

The long gestation, still longer nursing, and yet longer helpless period of the human emoryo and human infant demand the long nourishing care of the mother by the father and by the community. This means that for at least two years the father must provide food, protection and comfort, if not luxury, for the mother and child as well as for himself. If he fails in this duty, some other person, some charity, or the community must assume the duty. This being the fact, worthless, incapable and mentally or physically diseased fathers are a tax on the community.

All kinds of beautiful and necessary charities are succeeding in their aim to raise to adult life defective children, as well as to save the well and strong. A large number of these mentally, physically, and often through heredity, morally defective persons assert the right to marry, and do so, and another crop of defective children is the result. Our orphan asylums, hospitals, sanatoriums, homes, insane asylums, jails and prisons are being overfilled in consequence of the foregoing conditions. What are we going to do about it?

The environment of prospective fathers and mothers and their future children is being constantly improved by the public health advances now being made in all communities, but as has been stated, this will not prevent the ravages of inherited disease (syphilis; epilepsy, insanity, imbecility, physical weahness) or of the inherited tendency to disease (tuberculosis, cancer, gout, diabetes, alcoholism, etc.), any more than environment can produce twins, beauty, geniuses or permanent health. In fact, improved environment is doing more for the defectives in all lines than for those of good heredity, who would survive a less improved environment.

The question for discussion, then, in this one on eugenics is, who shall marry, and who shall have children? It seems doubtful whether the law or the state can, in the present status of the human age and of civilization, enforce scientific selection or compel complete physical and mental examination of a man and woman before they may be allowed to marry. Public health and welfare education, medical teaching and instruction, and the higher education emanating from the church may, in a few decades, make this problem one that is readily decided by the families of the contracting parties, or even by the young man and young woman most deeply interested. On the other hand, it is possible that the age is ripe for an insistence that known defectives and known diseased individuals be allowed to marry only when the other member of the contracting parties is made cognizant of the fact, and also that means be taken to prevent offspring from such alliances. Such defectives often breed readily and lavishly, and their progeny are often no comfort to themselves, and are more than likely to become a future, if not an immediate, tax on the community and state. This brings up the question of either prohibiting marriages of defectives (known to be such through public charities or public institutions and hence not ascertained by the compulsory examination of private individuals), or of legal sterilization of such defectives.

\section{THE SCHOOL QUESTION}

The following are a few suggestions of important subjects that medical men should impress privately on their families and publicly in their communities:

1. A child should be healthy before it goes to school (adenoids, tonsils, teeth, anemia, debility, etc.).

2. The kindergarten methods of teaching should be investigated, as some parts of this teaching are objectionable, and especially should the eyes of these young children not be overtaxed.

3. The best age for a child to attend a kindergarten is from 4 to 6 years; but the ordinary child at 6 years of age is too young to enter real school work. It would be ideal, from a physical point of view, if the child could then have one year, or in some instances two, in a "play school," combining physical exercise with moral and mental development. "Then the child would be well fitted to attend real school.

4. The location of school buildings is important, and the architecture and equipment of such buildings are very important.

5. There should be well-equipped playgrounds in all cities. 